\title{
Análisis de la aplicación de la gestión integral en seis empresas comerciales de tipo familiar en Florencia, Colombia
}

\section{Analysis of the application of the integral management in six commercial companies of family type in Florencia, Colombia}

\author{
Cristian Hernández-Gil ${ }^{1}$ \\ Beatriz Eugenia Vargas-Vargas ${ }^{2}$ \\ Beatriz Eugenia Gutiérrez-Mora ${ }^{3}$ \\ Edward Fabián Figueroa-Ramírez ${ }^{4}$ \\ Luis Eduardo Correa-Corrales ${ }^{5}$
}

Recibido: noviembre 06 de 2018

Aceptado: diciembre 28 de 2018

\section{Resumen}

El modelo de gestión integral se ha convertido en uno de los esquemas organizacionales más completos que reúne las áreas estratégicas de un ente económico, en función de tres componentes principales: la calidad, la seguridad en el trabajo y la gestión ambiental. Este documento tiene como objetivo presentar el análisis de la aplicación de la gestión integral en seis pymes comerciales de origen familiar, de Florencia, Colombia. El problema radica en la falta de competitividad y desarrollo empresarial de la región, lo que hace que no existan suficientes aspectos para darle credibilidad y valor a su economía. Se realizó un estudio de tipo descriptivo con enfoque cualitativo, donde se entrevistaron trabajadores de tres niveles jerárquicos (estratégico, táctico y operativo) de las seis pymes más reconocidas de Florencia. Los resultados indican que, a pesar de no existir un modelo de gestión integral explícito en las pymes de la región, sí se consideran algunos avances en su construcción, orientados bajo los siguientes elementos: la calidad en función del reconocimiento de marca, la aplicación de actividades de salud ocupacional, y los sistemas básicos de reciclaje.

Palabras clave: sistema de gestión, competitividad, empresa familiar, Pymes.

\begin{abstract}
The integral management model has become one of the most complete organizational schemes that gather the strategic areas of an economic entity, based on three main components: quality, job security and environmental management. The purpose of this paper is to present the analysis of the application of the integral management in six commercial companies of family origin, in Florencia, Colombia. The problem lies in the lack of competitiveness and business development of the region which makes the non-existence of enough aspects to give credibility and value to its economy. A descriptive study based on a qualitative approach was carried out, where workers from three hierarchical levels ( strategical, tactical, and operational) of the six most recognized commercial companies of family origin in Florencia were interviewed. The results indicate that despite the absence of an explicit integral management model for these companies in the region, some progress in their construction is considered, oriented under the following elements: quality based on brand recognition, application of occupational health activities and basic recycling systems.
\end{abstract}

Keywords: management system, competitiveness, family business, SMEs.

1 Administrador de Empresas, Especialista en Mercadeo Gerencial, Estudiante de Maestría en Ciencias de la Educación, Universidad de la Amazonia, Florencia, Colombia. E-mail: cris.hernandez@udla.edu.co

2 Ingeniera Industrial, Magíster en Administración, Organización Augusto Vargas Cardona, Florencia, Colombia. E-mail: gerencia.administrativa@drosur.com

3 Administradora de Empresas, Magíster en Administración, Caja de Compensación Familiar del Caquetá, Florencia, Colombia. E-mail: beatrizgutierrezm@hotmail.com

4 Estudiante de Administración de Empresas, Universidad de la Amazonia, Florencia, Colombia. E-mail: edw.figueroa@udla.edu.co

5 Estudiante de Administración de Empresas, Universidad de la Amazonia, Florencia, Colombia. E-mail: lui.correa@udla.edu.co 


\section{Introducción}

Toda empresa, ya sea por su tamaño, su nivel de ingresos, su patrimonio o inclusive por el número de trabajadores, debe desarrollarse en función del paradigma de la competitividad y la innovación. Para lograr esto, se ha iniciado dentro del mundo empresarial, la implementación de modelos de gestión integral que no solo implica certificarse en normas ISO de calidad, seguridad en el trabajo, y gestión ambiental, sino que, además, buscan fortalecer la cultura organizacional de cada unidad de negocio o ente económico (Sánchez, Hernández, Martínez, Villegas \& García, 2018). Colombia, siendo un país en donde casi el $98 \%$ del aparato productivo lo constituyen Pequeñas y Medianas Empresas, Pymes, donde el 95\% de ellas son familiares, no es ajena a esta realidad (Revista Dinero, 2016). Asimismo, las pymes en Colombia aportan cerca del 38\% del Producto Interno Bruto total, una cifra considerablemente baja teniendo en cuenta que en algunas economías desarrolladas estas pueden participar hasta en el $60 \%$ de la producción nacional (Revista Semana, 2015).

Según el Índice Departamental de Competitividad, IDC, determinado por el Consejo Privado de Competitividad, CPC, y la Universidad del Rosario (2015), el departamento del Caquetá se encuentra en la etapa 1 de desarrollo competitivo, ubicándose en el puesto 22 en la clasificación general, manteniendo por tres años consecutivos la misma posición. La medición se compone de 90 variables, agrupadas en 10 dimensiones o pilares de competitividad.

De los diez pilares que mide el índice, el mejor desempeño del Caquetá es en el pilar de medio ambiente (puesto 2), en el que el departamento escaló una posición con respecto al año anterior. Los desafíos más importantes en materia de competitividad en el departamento, se concentran en: tamaño de mercado, pilar en el que se ubica en la última posición (puesto 25), así como en educación básica y media, eficiencia de los mercados y sofisticación y diversificación, pilares en los que registra la penúltima posición (puesto 24).

Según el Plan de Desarrollo "Yo creo en Florencia" (Alcaldía de Florencia, 2016), sumado a los múltiples problemas sociales que generan un subdesarrollo económico complejo en el municipio, la corrupción y el "desgobierno" evidenciado durante los últimos años, y las medidas asistencialistas tomadas por el gobierno nacional, no han contribuido a proyectar económicamente a la ciudad capital del Caquetá. Esto se evidencia en: la falta de coherencia entre la oferta de educación y formación con relación a las oportunidades del mercado laboral, las pocas oportunidades laborales para los jóvenes profesionales, así como en las pocas iniciativas innovadoras y emprendedoras. Adicionalmente, la promoción de una cultura de alto consumo, donde el $87 \%$ de los productos requeridos en el mercado interno son traídos de otras ciudades, desestimulan la productividad y la creación de empleos dignos. Dentro de las 4.955 empresas constituidas ante la Cámara de Comercio de Florencia, CCF (2014), tan solo el 2,72\% de ellas desarrollan actividades profesionales, científicas o técnicas, lo cual refleja la deficiente capacidad innovadora y emprendedora de la ciudad.

Ante este panorama, es importante reconocer que la competitividad de la región sigue siendo un tema a tratar, el cual requiere el apoyo fundamental de la gestión tanto del sector público como del mismo sector privado y de la academia. Las empresas de Florencia presentan problemas de competitividad en sus unidades de negocio, que no les permite considerar el desarrollo de estrategias de diversificación y/o integración, quedando rezagadas a la utilización de tácticas de supervivencia y sin una planeación previa (Departamento Administrativo Nacional de Estadística, 2014).

El presente estudio tuvo como propósito indagar sobre la aplicación de la gestión integral en seis Pymes familiares comerciales de Florencia, para identificar sus esquemas de funcionamiento. La motivación que llevó a los investigadores a re- 
alizar este trabajo, se fundamenta en que la mayor parte de la economía del Caquetá, es producto de Pymes familiares; esto unido al bajo nivel de competitividad del Caquetá, que lo ubica de 22 entre los 25 departamentos analizados en Colombia. A continuación se consideran algunos elementos teóricos básicos para el desarrollo de este trabajo, que fue realizado entre los años 2016 y 2017. Luego se indica el alcance metodológico, se hace una interpretación de los resultados y la presentación de las respectivas conclusiones.

\section{Marco teórico y metodología}

\subsection{Competitividad y la gestión integral}

Tradicionalmente, los modelos de gestión usados en el mundo empresarial han estado enfocados en aspectos internos de la organización; sin embargo, la globalización de la economía ha llevado a mayores niveles de competitividad, que según González (2011), "obligan a considerar nuevos paradigmas y modelos de gestión que permitan adaptarse a las nuevas condiciones del entorno".

Las empresas de cualquier tamaño: micro, pequeña, mediana o grande, deben estar en capacidad de afrontar las circunstancias que se presentan en el entorno (influencia de otros sectores). Estas exigencias del mercado se materializan en variables de calidad y productividad de bienes y servicios, que exigen el diseño y ejecución de estrategias orientadas a garantizar su sostenibilidad (González, 2011). En la academia se dispone de un amplio menú de teorías que plantean nuevos paradigmas estratégicos y organizacionales, los cuales llevan a que la empresa deba evaluar cuál de todos implementar, de acuerdo a: sus necesidades, capacidades y requerimientos, dándole importancia a las que apunten a elevar su competitividad.

En este sentido, es necesario modernizar los procesos de gestión considerando las necesidades de las partes interesadas y los nuevos retos y riesgos, provenientes de las transformaciones propias de un contexto en continuo y acelerado cambio (Hernández-Gil, Figueroa-Ramírez \& Correa-Corrales, 2018). Así, se puede resaltar la importancia que han venido ganando los Modelos de Gestión Integral, MGl, como factor clave en la competitividad de las organizaciones. Los MGl incluyen aspectos relacionados con: la calidad de los bienes y servicios, ISO 9001, propuesta por la Organización Internacional para la Normalización, ISO (2008); la responsabilidad social hacia el ambiente, ISO 14001, homologada por el Instituto Colombiano de Normas Técnicas y Certificación, ICONTEC (2004); y la seguridad en el trabajo, ISO 18001 (ICONTEC, 2007), entre otras.

Las organizaciones son conscientes de que su capacidad de prosperar y crecer, depende de la existencia de una sociedad próspera y sostenible (Alean-Pico, del Río, Simancas-Trujillo \& Rodríguez-Arias, 2017). Igualmente, son conscientes de que las carencias sociales y la destrucción ecológica pueden tener impactos materiales negativos en la cadena de suministro, en los flujos de capital y en la productividad de los empleados (Fierro-Ulloa, 2013). Lo anterior ha permitido establecer un concepto tripartito, que involucra orientar los objetivos organizacionales hacia el bienestar de los clientes, al mejoramiento de la calidad de vida de los colaboradores de la empresa, así como al cuidado sostenible del ambiente.

En este contexto, Castillo y Martínez (2010), comparan las funciones del sistema respiratorio, al encargarse del suministro del oxígeno al corazón como un sistema prioritario de todo ser vivo, con el sistema de gestión de la calidad en la satisfacción del cliente, para garantizar: el equilibrio económico, su competitividad y los ingresos para las diferentes operaciones de cualquier organización como sistema abierto. Asimismo, para complementar la funcionalidad del sistema integral, la gestión en seguridad y salud ocupacional garantiza la disponibilidad de recurso humano libre de riesgos y sin enfermedades profesionales, mientras que el sistema de gestión ambiental apoya la protección y conservación del ambiente y pre- 
viene la contaminación, garantizando el suministro de materia prima en equilibrio con el entorno (Castillo \& Martínez, 2010).

Lo anterior implica que la gestión integral en las organizaciones juega un papel importante en el desarrollo de su competitividad. En este caso en particular, es menester que las empresas de la ciudad de Florencia, que en su mayoría son comerciales y de índole familiar, apliquen este tipo de esquemas para perdurar en el tiempo; de no hacerlo, quedarán rezagadas al fenómeno de la supervivencia organizacional, terminando a corto plazo en la parte final de su ciclo de vida: declive y muerte empresarial.

\subsection{Pymes familiares}

Las empresas familiares, según Pérez y Ramírez (2015), "son el motor de la economía de algunos países latinoamericanos; en Colombia representan el $95 \%$ del total del sector empresarial". Sin embargo, existen algunos problemas que enfrentan las empresas familiares, que aún hoy en día les impide cerrar la brecha de holgura para lograr la competitividad. Ejemplo de ello es la integración de los gastos familiares y particulares con los gastos de la organización; es decir, no hay diferenciación entre el ente empresarial y los gastos de la familia, constituyéndose en una amenaza que pone en riesgo la sostenibilidad empresarial (Arteaga-García \& Rodríguez-Jiménez, 2015). Esta situación surge por el poco control que se les da a los esquemas de planeación y organización internos, que permitan evidenciar un mejoramiento en la gestión de manera holística.

El estudio de las empresas familiares se apoya en el modelo de los círculos: dos círculos (Tagiuri \& Davis, 1982), tres círculos (Gersick, Davis, McCollom \& Lansberg, 1997) y cinco círculos (Amat, 2004). El modelo que ayuda a entender la gestión integral de las empresas familiares es el de cinco círculos, que supone cinco puntos importantes para la empresa: i) la familia, que relaciona los valores, actitudes y la relación intrafamiliar, en primero, se- gundo y hasta tercer grado de consanguinidad; ii) la propiedad, donde se consideran temas como: la estructura de poder y la correcta administración del patrimonio; iii) el negocio, es decir, los productos y servicios que ofrecen; iv) la gestión, es decir, la administración del recurso humano y tecnológico; y v) la sucesión, donde se trata de garantizar la continuidad de la empresa y de la familia empresarial (Amat, 2015).

El modelo de los cinco círculos inicia con la debida orientación hacia la relación familiar; es decir, la armonía en los flujos de comunicación y la justa ubicación de los miembros de la familia en los cargos que soporta la empresa. Cuando se logra esto, la empresa empieza a ser eficiente, pues adquiere una visión estratégica, tratando de lograr un mayor rendimiento con los productos y servicios ofrecidos. Los productos, a su vez, deben ser vendidos bajo parámetros que son determinados por la gestión, la cual se relaciona con: el grado de profesionalismo, control en los sistemas, las políticas de recursos humanos y el uso de la tecnología y la tecnificación de los procesos. En este punto se visualiza la gestión integral, la cual deja a un lado la naturaleza familiar del ente, enfocándose en la obtención de resultados, con miras a generar beneficios para todos los socios del negocio. Lo anterior es controlado por el líder, que generalmente es la cabeza de la familia, y quien usando la sucesión busca garantizar la continuidad de la empresa luego de su retiro.

En el contexto regional existe una alta presencia de Micro, pequeñas y medianas empresas de propiedad familiar. De acuerdo al Informe de Coyuntura Económica Regional Caquetá (DANE, 2014), se conformaron ante la CCF, 174 sociedades, con un capital de $\$ 8.262$ millones, las cuales lograron un crecimiento de $64 \%$ frente al año anterior; la actividad con mayor participación fue servicios con $39,3 \%$, seguida por transporte con $22,2 \%$. A su vez, ante la CCF se disolvieron 18 establecimientos, lo que conllevó a la reducción del patrimonio en \$1.978 millones; la actividad que más extrajo re- 
cursos fue el comercio con $\$ 1.008$ millones (CPC, 2016).

En cuanto a las Pymes familiares, no se tiene una estadística formal sobre ellas en la región. Según la Revista Semana (2015), en una encuesta realizada ese año por la firma Price Water House Coopers, PwC, a más de 2.400 empresas de familia colombianas, se reveló que solo el $16 \%$ de los consultados tiene un plan de sucesión discutido y documentado, mientras que a otras les cuesta mucho soltar el poder. Según el mismo estudio, el 32\% de las empresas familiares no quieren entregar el control a la siguiente generación. En las pymes la situación es más compleja. Por ejemplo, en Bancolombia el 98,5 por ciento de las más de 150.000 empresas que maneja la entidad son de familia y menos del $10 \%$ tienen protocolos formales establecidos. Son comunes los casos en algunas compañías donde: se suben los sueldos a discreción, se incurren en gastos para pagos personales o se vincula a familiares que no tienen las competencias para el cargo.

Uno de los estudios que más se acerca a entender la dinámica de gestión de las empresas familiares en la ciudad de Florencia, es el de Mora (2007). En cuanto al análisis de las etapas evolutivas, el autor concluye que: i) las empresas familiares son administradas y estructuradas con base en la unidad familiar, donde el fundador es el que manda y los demás miembros de la familia ocupan cargos de menor envergadura dentro de la empresa; ii) los puntos claves de éxito de estas empresas son sus fundadores, quienes se convirtieron en los pioneros, con deseos de surgir, gastando estrictamente lo necesario, hacen lo que les gusta, le dan valor a la palabra siendo esta señal de credibilidad, trabajan arduamente, ofrecen exclusividad y excelente servicio, adicionalmente las ideas de negocios son materializadas; iii) las empresas incursionan, adaptándose al cambio, sosteniéndose y buscando el incremento de su participación en el mercado; iv) el talento humano es considerado el activo más valioso, por ello se le capacita constantemente en busca del beneficio para la empresa; v) el manejo financiero está cargo del fundador.
En cuanto a los sistemas de gestión integral en las empresas familiares, Mora (2007), aclara que las empresas de Florencia basan su gestión en el empirismo de sus dueños, pioneros en hacer las cosas por pasión y buscando siempre oportunidades para hacer de todo un negocio, un emporio honesto y trascendental que pueda ser promovido en el tiempo por las nuevas generaciones.

\subsection{Metodología}

La investigación tuvo un enfoque cualitativo que permitió caracterizar las variables que componen un Modelo de Gestión Integral, MGl, así como desarrollar un esquema estratégico que reconozca la contribución de este modelo en la competitividad de las pymes familiares de Florencia. Un investigador cualitativo prefiere que la teoría emerja de los propios datos (Cook \& Reichard, 1986). Esta cimentación de la teoría de los datos incrementa la capacidad del investigador para comprender y quizá para concebir una explicación del fenómeno, que sea consecuente con su aparición en el mundo social (Muñoz-Saravia, 2007).

Igualmente, se realizó un estudio de tipo descriptivo, el cual trata de detallar las características más importantes de un determinado objeto de estudio con respecto a su aparición y comportamiento, buscando la mejor manera de caracterizar su realidad en una situación o contexto dado (Hernández, Fernández \& Baptista, 2015). Este estudio es una investigación aplicada, que confronta la teoría correspondiente a los sistemas integrados de gestión, con la realidad de la gestión de las pymes familiares de la ciudad de Florencia, enfocada en aspectos de calidad, medio ambiente y seguridad en el trabajo.

El instrumento para la recolección de información fue un cuestionario estructurado, cuya técnica fue la entrevista a profundidad. Vargas-Jiménez (2012), reconoce que esta técnica tiene como fin obtener información de los participantes, fundamentada en: las percepciones, las creencias, las 
opiniones, los significados y las actitudes, desde su experiencia en la organización donde laboran.

El muestreo fue no probabilístico, de tipo intencional, que parte de la elección de los elementos muestrales de una serie de criterios, que se consideran necesarios o altamente convenientes para tener una unidad de análisis con las mayores ventajas para los fines que persigue la investigación (Martínez, 2004, citado por Arenas-Cardona \& Rico-Balvín, 2014). Los criterios utilizados por los investigadores en este trabajo, fueron: empresas con más de 10 años de labor en el mercado de Florencia, Caquetá; empresas que pertenecen al sector comercial y que sean de origen familiar; empresas con voluntad para ser parte del conjunto de sujetos analizados (permiso para utilizar información suministrada). Inicialmente se perfilaron 10 empresas para el estudio, pero solo seis de ellas aceptaron participar. Es necesario resaltar que, según el último criterio establecido, se llegó a un acuerdo de confidencialidad con las seis empresas escogidas de no revelar su información corporativa; es decir, su razón social y sujetos entrevistados.

En total se realizaron 9 entrevistas por cada empresa, de modo que la unidad de trabajo la conformaron 54 personas, todas de cargos en diferentes niveles jerárquicos, para llegar a entender la mecánica del modelo de gestión integral de manera holística. Cada entrevista se interpretó desde la intencionalidad, para la cual Castillo y Martínez (2010), establecen que la conducta humana no es un mero acto o movimiento físico, tiene una meta y está animada por una intención; debido a ello, comprender una conducta humana es percibirla, de algún modo, desde adentro, desde el punto de vista de la intención que la anima. El análisis de los datos se realizó con el Software Atlas. Ti, estableciendo tres categorías de análisis: calidad de los Productos; seguridad en el Trabajo; gestión Ambiental y sus relaciones entre los puntos de convergencia y divergencia, de los seis sujetos investigados, por cada unidad de negocio.

La validez y confiabilidad de los instrumentos se identificó teniendo en cuenta las siguientes fases: i) Inicialmente con el cuestionario piloto se realizaron nueve entrevistas a dos empresas seleccionadas en la muestra, esto para validar la teoría y evidenciar qué cambios se le podían hacer al instrumento. ii) Seguidamente, se realizaron las modificaciones respectivas ejecutando otras nueve entrevistas (dos nuevas empresas), y así se hicieron nuevos cambios al instrumento. iii) Se finalizó con las dos empresas restantes y el instrumento quedó completamente perfeccionado.

\section{Resultados y discusión}

3.1 Planeación, toma de decisiones y direccionamiento estratégico

En la Tabla 1 se presentan los grupos de códigos, los códigos o categorías y los memos, de cada red, las cuales se construyeron de acuerdo a los elementos del sistema de gestión integral. 
Tabla 1. Elementos de análisis para la aplicación de datos en software Atlas. Ti

\begin{tabular}{|c|c|c|c|}
\hline Redes & $\begin{array}{l}\text { Gupos de } \\
\text { códigos }\end{array}$ & Códigos o categorías & Temas de las citas \\
\hline \multirow{3}{*}{$\begin{array}{l}\text { RED No. 1: } \\
\text { Calidad }\end{array}$} & \multirow{3}{*}{$\begin{array}{l}\text { Componente } \\
\text { Calidad }\end{array}$} & $\begin{array}{l}\text { Direccionamiento es- } \\
\text { tratégico }\end{array}$ & $\begin{array}{l}\text { - Sistema de planeación vigente } \\
\text { - Participantes en la planeación } \\
\text { - Planes alternos (comercial) } \\
\text { - Modelo de negocio ajustado a franquicia } \\
\text { - Modelo de negocio }\end{array}$ \\
\hline & & Gerencia de procesos & $\begin{array}{l}\text { - Características del modelo de negocios } \\
\text { - Planes y manuales de funciones } \\
\text { - } \quad \text { Modelo de franquicia de marcas reconocidas } \\
\text { - } \quad \text { Análisis de la distribución como calidad. }\end{array}$ \\
\hline & & (1) & $\begin{array}{l}\text { - Control de fijación de precios } \\
\text { - } \quad \text { Calidad desde el reconocimiento de marca y } \\
\text { contenido del producto. } \\
\text { - Estrategia de distribución } \\
\text { - Estrategia de segmentación } \\
\text { - Diversificación de marcas } \\
\text { - Marcas tradicionales } \\
\text { - Motivación implícita }\end{array}$ \\
\hline $\begin{array}{l}\text { RED No. 2: Salud } \\
\text { y seguridad en } \\
\text { el trabajo }\end{array}$ & $\begin{array}{l}\text { Componen- } \\
\text { te Gestión y } \\
\text { seguridad en el } \\
\text { Trabajo }\end{array}$ & $\begin{array}{l}\text { Gestión de Seguridad en } \\
\text { el trabajo }\end{array}$ & $\begin{array}{l}\text { - Asignación de funciones } \\
\text { - Motivación salarial } \\
\text { - Planes de salud ocupacional } \\
\text { - Actividades de salud ocupacional } \\
\text { - Sistema de reciclaje }\end{array}$ \\
\hline $\begin{array}{l}\text { RED No. 3: } \\
\text { Gestión ambi- } \\
\text { ental }\end{array}$ & $\begin{array}{l}\text { Componente } \\
\text { Gestión Ambi- } \\
\text { ental }\end{array}$ & Gestión reciclaje & $\begin{array}{l}\text { - Conocimiento de leyes } \\
\text { - Conocimiento de impactos sobre el ambiente } \\
\text { - Responsabilidad frente al ambiente }\end{array}$ \\
\hline
\end{tabular}

En general, el análisis de los datos indica que no existe ningún proceso continuo o certificación definida en términos de calidad, seguridad para el trabajo o gestión ambiental, en las empresas objeto de estudio. Sin embargo, es importante con- siderar que sí existe la aplicación de un Modelo de Gestión Integral, MGl, parcialmente definido y de manera implícita dentro de las actividades diarias de cada ente. 
En cuanto al sistema de planeación, toma de decisiones y direccionamiento estratégico vigente, se identificaron las siguientes características:

Tanto los directivos del nivel estratégico como los colaboradores del nivel táctico, a pesar de que argumentan la misión y la visión de su organización, no lo hacen de manera literal como están establecidas y adicional a ello el enfoque que le dan es solo interno. Es decir, para ellos en la misión, lo importante es "la comercialización de productos de calidad entregados en el tiempo establecido". Pero no hay una consideración de la participación del consumidor o cliente y su satisfacción, principal elemento de la calidad en cualquier sistema integral (López-Celis \& Peñalosa-Otero, 2017).

Los colaboradores del nivel operativo no reconocen la misión y la visión de la organización cuando se les preguntó. Algunos de ellos no están familiarizados o no conocen estos dos conceptos. Actualmente, en ninguna de las empresas existe una programación explícita para el desarrollo de un proceso planificador para cada año futuro. Para los directivos, la planeación es el desarrollo de un plan comercial o de ventas que no es consecutivo año tras año.

Los encargados de los niveles tácticos y operativos aseguran que no participan dentro del proceso de planeación y mucho menos en la toma de decisiones, la cual está en cabeza de los mismos dueños o gerentes de la empresa. A pesar de que la toma de decisiones es de manera centralizada, en las empresas se evidencia cierta dependencia con la organización que les adjudica el permiso para comercializar los productos de su marca.

Las razones dadas por los encargados del nivel directivo, respecto a la no participación en la toma de decisiones de los colaboradores de nivel táctico y operativo, son: porque la planeación depende de las exigencias del contrato establecido con la multinacional que da los permisos para la comercialización de productos de la marca. Además, consideran que los cargos del nivel táctico no tienen ese tipo de funciones dentro de su manual de procesos u operaciones.

\subsection{Estado del modelo de gestión integral}

\section{Gestión de la calidad}

Actualmente ninguna de las empresas cuenta con una certificación de calidad o similar, que permita evidenciar la importancia que el ente le da a este componente. La calidad, en la mayoría de los casos, es entendida bajo dos aspectos: el cumplimiento de las obligaciones salariales con los empleados o colaboradores, y las características tangibles e intangibles del producto.

Lo anterior permite distinguir que, analizando el modelo, desde la visión teórica, no se establece una clara relación o coherencia entre los aspectos que realmente deberían ser considerados dentro de la calidad, como lo son: la satisfacción del consumidor, la gestión de procesos y la tecnología y la gestión del talento humano.

Asimismo, según los entrevistados, ninguna empresa considera para los próximos años la vinculación de un plan de certificación en alguna norma de calidad. Las razones expuestas son de tipo enunciativo: no es necesario para el desarrollo del objeto social, no ha sido una exigencia de las empresas con las marcas que se comercializan, no son empresas manufactureras. En la figura 1 se reflejan la red semántica y las relaciones entre códigos de este componente del modelo. 


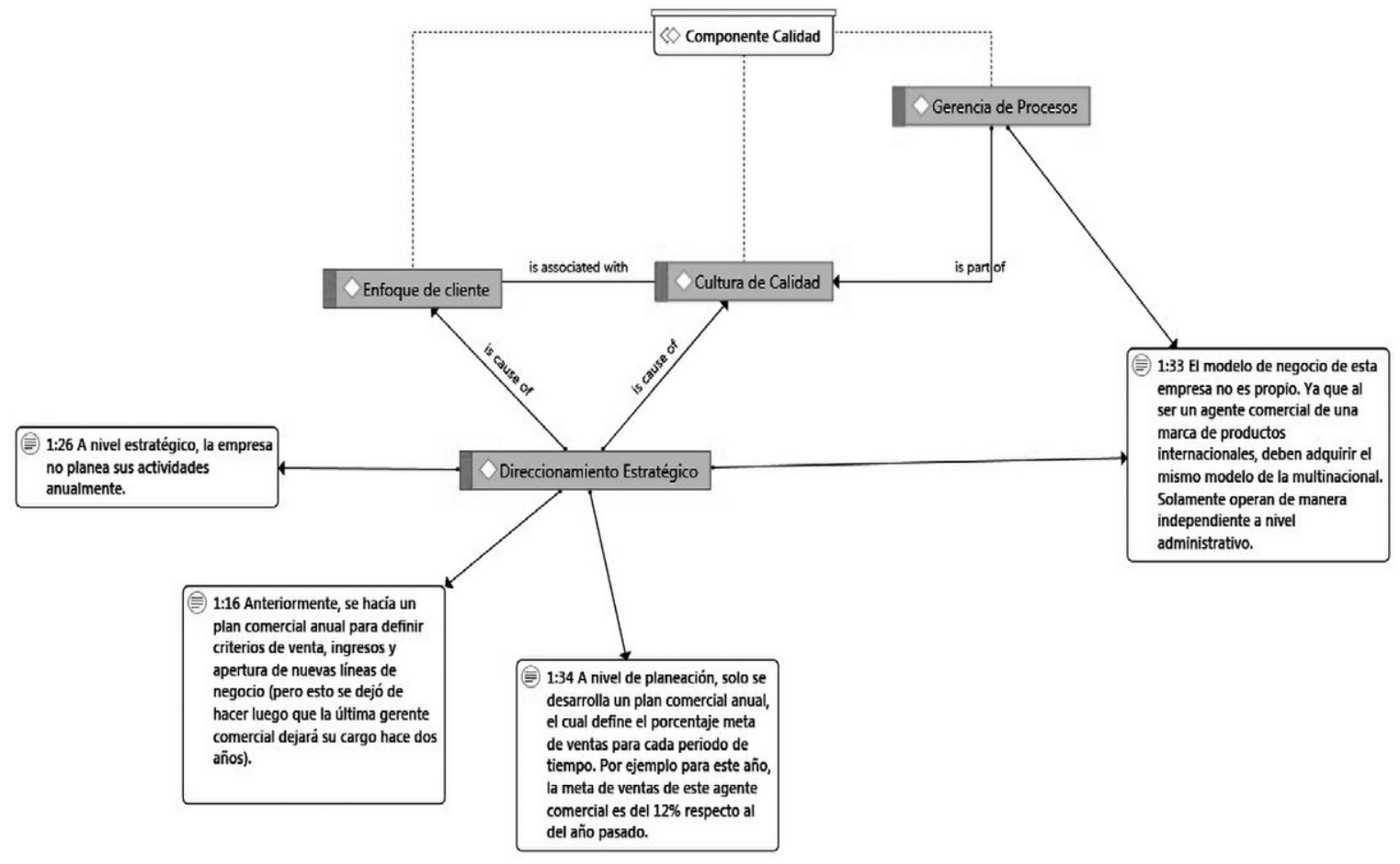

Figura 1. Red semántica del componente Gestión de la calidad.

Adicionalmente, ninguno de los funcionarios entrevistados expresó de manera literal la misión y la visión de la empresa. Solo los encargados de los niveles tácticos y estratégicos parafrasearon estos dos elementos del direccionamiento estratégico, descuidando el más importante: la satisfacción del cliente.

\section{Gestión de la seguridad en el trabajo}

A nivel de este componente de la gestión integral, no se evidencia mayor desarrollo. Las seis empresas han subcontratado los servicios de agencias dedicadas a la elaboración y asesoría en la aplicación de planes de salud ocupacional. En este momento, las empresas cuentan con el plan a través de un documento físico, pero solo tres de ellas han empezado a ejecutarlo. Las empresas que no han puesto en marcha la aplicación del plan, se enfrentan a circunstancias relacionadas con falta de tiempo y la ausencia de un departamento formal, que realice actividades de salud ocupacional con los empleados.
Asimismo, existe un nivel alto de desmotivación de los empleados de nivel táctico y operativo, con respecto a su participación en estos planes, debido a que ellos no desean ceder parte de su tiempo de descanso o entretenimiento para la realización de actividades de salud ocupacional. Adicionalmente, los empleados no están usando de manera adecuada y constante las herramientas de trabajo, especialmente aquellos en los que los riesgos por problemas de salud son mayores. Ejemplo de ello son los que trabajan en zona de descargue y cargue de mercancía, en áreas como la bodega, o aquellos que se encargan de la manipulación de elementos nocivos (productos de aseo), que también son comercializados.

\section{Gestión ambiental}

Este componente también ha sido mal enfocado por parte de las empresas objeto de estudio, debido a que sus empleados o colaboradores afirman que desde la gestión empresarial no se generan impactos negativos al ambiente, por no ser em- 
presas manufactureras. De esta manera, no cuentan con una certificación de prácticas de responsabilidad social o no existe un plan ejecutado, que mida y mitigue algunos impactos en materia de generación de basuras y desperdicios.

La gestión ambiental es entendida como el desarrollo de sistemas básicos de reciclaje, especialmente de papel y cartón, los dos principales elementos que utilizan las empresas estudiadas. Sobre lo anterior, se plantean dos opciones: En primera medida, el reciclaje de estos materiales y la venta posterior de los mismos; en segunda medida, la reutilización -sobre todo del cartón-, en el empaque de mercancía no fabricada por la empresa, pues el manejo actual no evidencia una cultura de conciencia ambiental. En la Figura 2 se ilustra la red semántica de este componente.

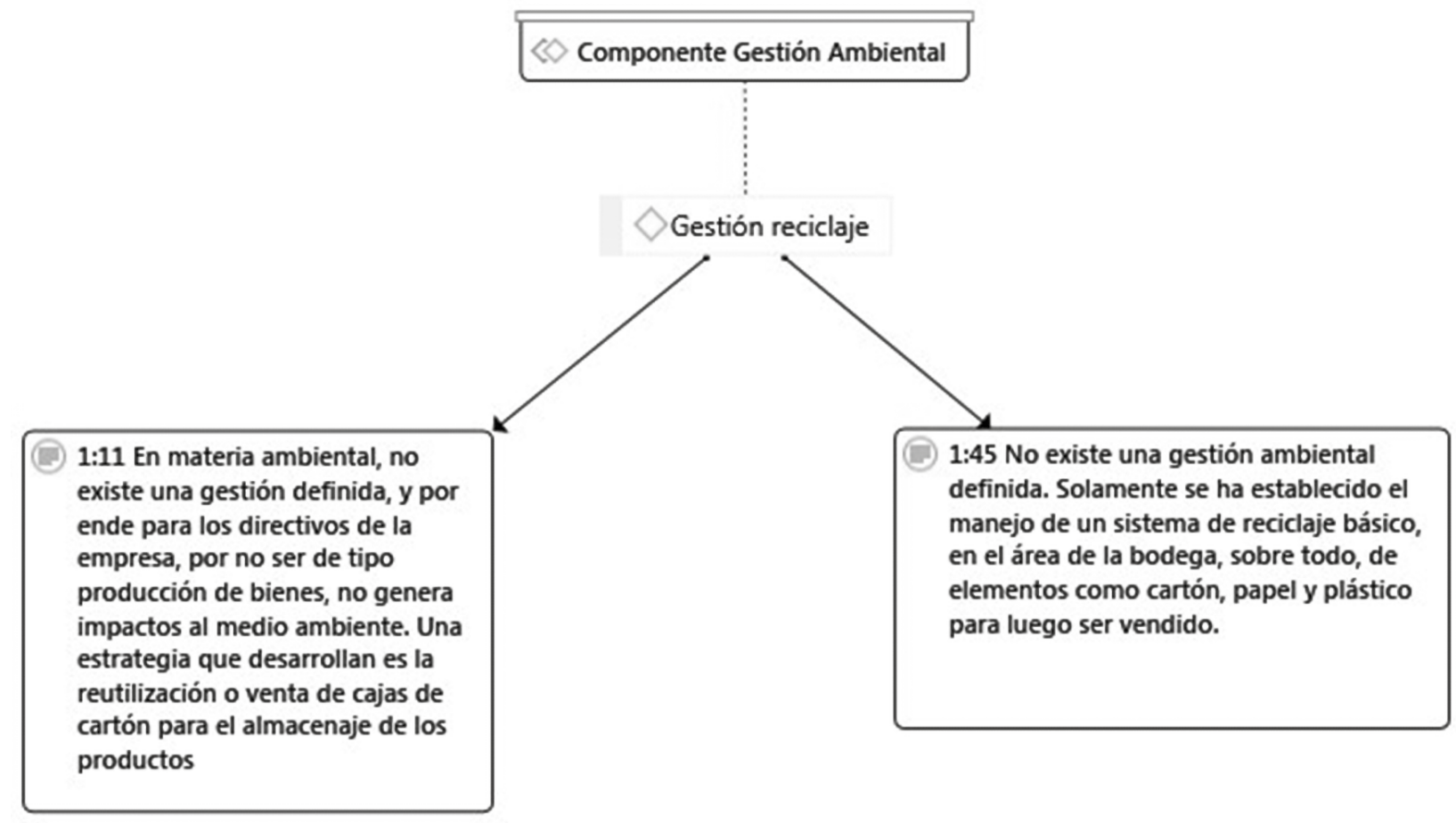

Figura 2. Red semántica del componente Gestión Ambiental.

En la Tabla 2 se resumen los elementos del Modelo de Gestión Integral, MGl, implícitamente aplicados por las empresas objeto de estudio. Es necesario tener en cuenta que de los 7 elementos identificados: cuatro de ellos pertenecen al eje de la gestión de la calidad; dos a la Gestión en Seguridad en el trabajo y un solo elemento en el eje de Gestión Ambiental, ya que es el componente con menos desarrollo en la gestión empresarial de las Pymes comerciales familiares de la ciudad de Florencia. 
Tabla 2. Elementos del MGl identificados en las empresas estudiadas.

\begin{tabular}{|c|c|c|}
\hline Eje del sistema & Elemento & Estado del arte \\
\hline & $\begin{array}{l}\text { Direccionamiento } \\
\text { estratégico }\end{array}$ & $\begin{array}{l}\text { Todas las empresas cuentan con misión y visión, enmarcadas en un di- } \\
\text { reccionamiento estratégico, donde la falta de procesos de planeación } \\
\text { ha hecho que estas queden obsoletas en el tiempo sin tener en cuenta } \\
\text { los retos y cambios del mercado local, regional y nacional. Los directivos } \\
\text { conocen la misión y la visión, aunque son muy pocos los que la llegan } \\
\text { a recitar al pie de la letra; además, se refleja ausencia de los elementos } \\
\text { que deben tener estas, de acuerdo a la literatura administrativa. }\end{array}$ \\
\hline
\end{tabular}

En su mayoría los procesos están liderados en el diario funcionamiento del negocio. A pesar de que existe manual de procedimientos y de funciones de cada cargo, estos son presentados como desactualizados o en Gerencia de procesos proceso de perfeccionamiento. De ahí que cada proceso se realice por la costumbre habitual del desarrollo del objeto social de la empresa. Los directivos afirman conocer sus procesos, y aseguran que el liderazgo se hace de manera centralizada, y solo los dueños son los encargados de

Calidad tomar decisiones para el futuro o bienestar de cada pyme.

El concepto de calidad para todas las pymes se concentra en el reconocimiento de marca de los productos que venden a través de sus canales de distribución y de acuerdo a la estrategia de segmentación definida.

Cultura de calidad La calidad es entendida como el trabajo bien hecho, sin errores, sin desperdicios de tiempos y movimientos. Además, una cultura de calidad en estos lugares se percibe por la motivación extrínseca orientada a sus empleados (pagos salariales oportunos) y la satisfacción de sus clientes (tiempo de entrega de acuerdo a lo prometido en cada pedido).

Existe un enfoque orientado hacia el cliente toda vez que la calidad es

Enfoque hacia el cliente

Gestión del talento humano

Seguridad para el trabajo

Gestión de seguridad en el trabajo

Gestión ambiental vista como la comercialización de productos de marcas reconocidas. Solo dos empresas (agentes comerciales de marcas reconocidas en el país) a través de una cultura enfocada en la satisfacción del cliente externo e interno son las que llevan un enfoque guiado hacia el cliente.

La gestión del talento humano, tiende a ser confundida con el componente de la calidad. Existen manuales de funciones para cada cargo en proceso de actualización. Y la motivación hacia el personal tiene dos consideraciones: la remuneración a tiempo y la celebración de eventos empresariales internos en lugares diferentes a la organización.

Se establece la subcontratación de la asesoría, realización y aplicación de un plan de salud ocupacional para todo el negocio. Los planes para todas las empresas actualmente están en proceso de ejecución. Y existe dos dificultades que han impedido su desarrollo completo: 1) la ausencia de un departamento de salud ocupacional que apoye la gestión del área de talento humano. 2) la controversia entre empleados y directivas de la realización de actividades de salud ocupacional en horas diferentes a las laborales.

Es el elemento menos desarrollado hasta el momento. $Y$ en todos los casos lo asocian al desarrollo de un sistema de reciclaje básico, especialmente de reutilización o venta de papel y cartón. Los directivos y demás colaboradores, aseguran que, por no ser una empresa manufacturera, no existen impactos ambientales negativos. $Y$ a nivel de responsabilidad social, es entendido como actividades de donaciones para celebraciones de fin de año a familias o personas con necesidades básicas insatisfechas. 


\subsection{Discusión}

El término competitividad en las organizaciones estudiadas, es ilustrado no como la capacidad para permanecer en el mercado sino como el enfrentamiento de retos que impone el mercado a través de la implementación de estrategias de la competencia, para lograr vender más. Además, todas las empresas, al respecto, argumentaron las mismas dificultades, desconocimiento y las pocas acciones sobre el tema, las cuales se sintetizan a continuación:

i) La competitividad de las pymes analizadas está enfocada en función de la marca de los productos que ellos comercializan, con gran reconocimiento a nivel nacional e internacional. A nivel de calidad, la competitividad se manifiesta en la relación con los proveedores, multinacionales de marcas de gran trayectoria en el mercado nacional y latinoamericano. Por ejemplo, dos empresas que son agentes comerciales, distribuidores exclusivos de marcas con buen posicionamiento en el mercado, afirman que esa es su ventaja competitiva, al no haber más competidores que manejen la distribución de la misma marca en la zona.

ii) A nivel de gestión del talento humano, no se percibe algún hecho competitivo. Para los directivos -nivel estratégico-, la competitividad se percibe desde la remuneración oportuna a sus colaboradores -niveles tácticos y operativos-, y a la integración social entre todos los empleados de la empresa en lugares diferentes a la organización.

iii) A nivel de gestión ambiental, no se evidencia ningún hecho que genere competitividad. Actualmente ninguna de las empresas analizadas hace parte de alguna red de competitividad o comisión regional para la competitividad. En su mayoría las empresas analizadas expresan su poco interés en ser parte de estos comités o agremiaciones, dando una respuesta que se resume en la afirmación de uno de los gerentes entrevistados: "si no somos competitivos desde nuestro negocio, ¿cómo vamos a promover dicha competitividad a nivel regional?, primero lo primero".

En relación con otras investigaciones, se denota una significativa brecha de desarrollo competitivo. En el estudio de Cote-Peña, Meneses-Amaya, Arenas-Morantes y Caballero-Pérez (2016), se perfila al departamento de Santander como altamente competitivo, debido al mejoramiento de los niveles de inversión en actividades científico tecnológicas que han incrementado las innovaciones de las empresas, a través de los Centros de Desarrollo Productivo, CDP. En este caso, se le da importancia al apoyo del sector público para el desarrollo competitivo de las organizaciones, circunstancia que no es tenida en cuenta por las empresas estudiadas en la ciudad de Florencia; ellas, en su mayoría, se escudan en la comercialización de marcas ajenas con respaldo internacional, lo que significa bajos niveles de innovación propios. Por ello, una de las conclusiones del estudio en Santander revela que la consolidación de una cultura innovadora, podría lograr la competitividad empresarial y regional, si y solo si las empresas, desde su gestión financiera, asignan presupuestos adecuados para la inversión en actividades de investigación y desarrollo. En este orden de ideas, el sistema de gestión integral cobrará vida en las empresas familiares de Florencia cuando, dentro de sus principios corporativos, la innovación sea respaldada por acciones que produzcan bienes y servicios únicos y diferenciados.

Asimismo, la investigación de Morales-Rubiano, Ortiz-Riaga, Duque-Orozco y Plata-Pacheco (2017), sobre la competitividad del sector turístico de Bogotá y Pereira, reconoce un elemento importante que las organizaciones familiares de Florencia no han tenido presente dentro del desarrollo de su objeto social: la gestión del conocimiento. Los autores definen el conocimiento como un factor estratégico que implica la generación de capacidades para la innovación a favor del crecimiento y la competitividad. Entre las fuentes de 
conocimiento se resalta el papel de los empleados, los cuales están implicados directamente en el servicio o en el contacto con el cliente. Es decir, existe un verdadero valor para generar competitividad desde los niveles tácticos y operativos, que no lo perciben las empresas familiares en Florencia, donde todo proceso de decisión es centralizado por el nivel estratégico.

Hernández-Fuentes y Sánchez-Mojica (2017), en su investigación sobre la relación entre la competitividad y la innovación en las empresas del sector agroindustrial en Cúcuta, consideran que las empresas que más innovan pueden ser las más competitivas porque están a la vanguardia de los procesos y técnicas para desarrollar de manera eficiente su actividad productiva. En este sentido, existe una relación directa y positiva entre la innovación y la competitividad, explicada en: un aumento del uso de tecnologías, la reorganización de la empresa en áreas administrativas y operativas a fin de establecer la importancia de la mejora continua, y un aumento competitivo reflejado en un mayor número de ventas, de los ingresos y por ende de los beneficios percibidos. Sin embargo, el panorama de las empresas familiares de Florencia es similar al de las microempresas del sector agroindustrial de Cúcuta, las cuales evidencian una falta de mejora en la capacidad de innovar en el proceso, producto y organización empresarial, a fin de contribuir a la competitividad de la ciudad. Esto se soporta en que el \%11 de las empresas encuestadas en Cúcuta, no realizó ninguna innovación en los últimos 5 años, el \%48 no tiene ningún acuerdo comercial con otras empresas y el \%32 no tiene contacto con las agremiaciones empresariales. En el caso de la investigación aquí reportada, se refleja la nula participación de las empresas en comités regionales de competitividad e inclusive la inexistencia de convenios o alianzas con otras organizaciones, para alcanzar el paradigma de la competitividad.

Las empresas, no importa su tamaño o su nicho de negocio, deben ser capaces de perdurar y ser autosostenibles en el tiempo, a partir de una buena gestión; para ello, es importante utilizar o implementar un modelo de negocio que brinde la oportunidad de sobrevivir en el largo plazo (Vergara-Arrieta, 2015; Cardona-Arbeláez, Alzate-Alvarán \& Lora-Guzmán, 2018). En este sentido, el conocimiento adquiere una nueva interpretación, y es la de entender una realidad de acuerdo al contexto en el que se desenvuelve la organización (Del Río-Cortina, Cardona-Arbeláez \& Guacarí-Villalba, 2017). No todos los modelos se pueden adaptar a todas las empresas, así como todas las empresas no pueden lograr desarrollar un modelo si no están lo suficientemente organizadas para trabajar en función de los resultados. De ahí que comprender la totalidad de una empresa y su aplicación de la gestión integral, es una tarea dispendiosa y compleja, que requiere la realización de un trabajo específico y personalizado; este trabajo implica analizar, aspecto por aspecto, las condiciones para que la empresa pueda adaptarse a un modelo específico.

\section{Conclusiones}

Luego de hacer el análisis individual y colectivo, contrastándolo con la evidencia teórica, se presentan algunas omisiones de los entes económicos que participaron en el estudio. Por ejemplo, las empresas, dentro de su desarrollo, no cuentan con un modelo de gestión integral diseñado e implementado para el desarrollo de su objeto social. Además, no tienen claro el modelo de direccionamiento estratégico, pues trabajan persiguiendo únicamente el objetivo comercial o económico, sin considerar otros aspectos relevantes como la responsabilidad social como objetivo ético.

En otro sentido, se percibe que existen algunos elementos propios de cada aspecto que conforman un modelo de gestión integral, pero desarrollados de manera implícita; es decir, no son reconocidos por los actores organizacionales como parte de un modelo de gestión. A nivel de calidad, se trabaja en función del proceso de venta, características tangibles e intangibles de los productos. En cuanto al aspecto seguridad en el trabajo, están desarrollan- 
do manuales de salud ocupacional con asesoría externa subcontratada, en aras del cumplimiento legal. En el último aspecto, el de responsabilidad ambiental, se considera que no es un tema de mucha importancia, pues se evidenció la realización de pocos procesos de reciclaje.

En este análisis se encontraron aciertos y desaciertos en el diseño, implementación y ejecución de un modelo de gestión integral. Las empresas objeto de estudio no están en condiciones de certificarse para desarrollar los elementos del modelo a través de las normas ISO. Y esto se debe a la infraestructura de cada empresa y a la mentalidad estratégica de la familia que direcciona la misma. También se destaca que los elementos del modelo de gestión integral no están conectados o alineados al deber ser -según la literatura gerencial-, de un modelo de estas características, primero por desconocimiento de sus directivos y segundo por la concentración de esfuerzos en un solo proceso: la venta.

Finalmente, se puede concluir que las empresas analizadas no desarrollan estrategias para mejorar su competitividad. Además, se denota un interés nulo por ser partícipes del mejoramiento de la competitividad regional. Para futuras investigaciones queda abierto el interrogante de: ¿Cómo constituir y consolidar una cultura de innovación, competitividad y gestión integral en las empresas pequeñas y medianas de la región del Caquetá?.

\section{Referencias}

Alcaldía de Florencia (2016). Plan de desarrollo municipal "Yo creo en Florencia: seguridad, infraestructura, empleo" 2016-2019. Florencia, Colombia.

Alean-Pico, A., Del Rio, J., Simancas-Trujillo, R., \& Rodríguez-Arias, C. (2017). ¿El emprendimiento como estrategia para el desarrollo humano y social?. Saber, Ciencia Y Libertad, 12 (1). Recuperado de: http://www.sabercienciaylibertad.org/ojs/index.php/scyl/article/view/218
Amat, J. M. (2004). La continuidad de la empresa familiar. Barcelona, España: Gestión 2000.

Arenas-Cardona, H. A., \& Rico-Balvín, D. (2014). La empresa Familiar, el protocolo y la Sucesión Familiar. Estudios Gerenciales, 30 (132), 252-258. Recuperado de: http://www.scielo.org.co/pdf/eg/ v30n132/v30n132a06.pdf

Arteaga-García, M. E., \& Rodríguez-Jiménez, L. A. (2015). Análisis de los modelos de empresas familiares. (Thesis de maestría). Universidad de Especialidades Espiritu Santo. Recuperado de: http:// repositorio.uees.edu.ec/handle/123456789/2302

Cámara de Comercio de Florencia, CCF. (2014). Análisis de la Situación Económica de Caquetá. Florencia, Colombia.

Cardona-Arbeláez, D., Alzate-Alvarán, J. C., \& Lora-Guzmán, H. (2018). Estrategias para la gestión del talento humano en las asociaciones de yuqueros adscritas a Colfeyuca en Sucre, Colombia. Revista de Investigación, Desarrollo e Innovación, 9 (1), 9-18. doi: https://doi.org/10.19053/20278306. v9.n1.2018.8501

Castillo, D., \& Martínez, J. (2010). Enfoque para Combinar e Integrar la Gestión de Sistemas. Bogotá, Colombia: ICONTEC.

Consejo Privado de Competitividad., \& Universidad del Rosario (2015). Índice Departamental de Competitividad 2015. Bogotá, Colombia. Recuperado de: https://compite.com.co/wp-content/uploads/2016/05/CPC_-Resumen-2015-2016.pdf

Consejo Privado de Competitividad, CPC. (2016). Informe Nacional de Competitividad 2016-2017. Bogotá, Colombia. Recuperado de: https://compite. com.co/wp-content/uploads/2016/11/CPC_Libro_Web_2016-2017.pdf 
Cook, T., \& Reichardt, C. (1986). Métodos cualitativos y cuantitativos en investigación evaluativa. Madrid, España: Morata S.L.

Cote-Peña, C. P., Meneses-Amaya, C. P., Arenas-Morantes, C. J., \& Caballero-Pérez, D. I. (2016). Benchmarking entre sistemas regionales de innovación: el caso de Santander y Antioquia, Colombia. Revista de Investigación, Desarrollo e Innovación, 7(1), 1124. doi: 10.19053/20278306.v7. n1.2016.4088111

Del Río-Cortina, J., Cardona -Arbeláez, D., \& Guacarí-Villalba, A. (2017). Responsabilidad social empresarial y construcción de la marca: una nueva mirada a las estrategias de gestión. Revista de Investigación, Desarrollo e Innovación, 8 (1), 49-60. doi: https://doi.org/10.19053/20278306. v8.n1.2017.7370

Departamento Administrativo Nacional de Estadística, DANE. (2014). Informe de Coyuntura Económica Regional. Colombia: Banco de la República. Recuperado de: http://www.banrep. gov.co/sites/default/files/publicaciones/archivos/ icer_caqueta_2014.pdf

Fierro-Ulloa, I. (2013). Comportamiento organizacional positivo: implicaciones para la organización actual. Saber, Ciencia y Libertad, 8 (2), 103111. Recuperado de: https://dialnet.unirioja.es/ servlet/articulo?codigo $=5104984$

Gersick, K. E., Davis, J. A., McCollom Hampton, M., \& Lansberg, I. (1997). Generation to Generation: Life Cycles of the Family Business. Boston, USA: Harvard Business Press

González, S. (2011). Sistemas Integrados de gestión, un reto para las pequeñas y medianas empresas. Escenarios, 9 (1), 69-89. Recuperado de: https://uac.edu.co/images/stories/publicaciones/ revistas_cientificas/escenarios/volumen-9-no-1/ art07.pdf
Hernández, S., Fernández, C., \& Baptista, L. (2015). Metodología de la Investigación. México D.F.: McGraw Hill.

Hernández-Fuentes, S. N., \& Sánchez-Mojica, K. Y. (2017). Innovación y competitividad: micro y pequeñas empresas del sector agroindustrial en Cúcuta. Revista de Investigación, Desarrollo e Innovación, 8 (1), 23-33. doi: 10.19053/20278306.v8. n1.2017.736823

Hernández-Gil, C., Figueroa-Ramírez, E. F., \& Correa-Corrales, L. E. (2018). Reposicionamiento de marca: el camino hacia la competitividad de las pequeñas y medianas empresas. Revista de Investigación, Desarrollo e Innovación, 9 (1), 33-46. doi: https://doi.org/10.19053/20278306. v9.n1.2018.8505

Instituto Colombiano de Normas Técnicas y Certificación, ICONTEC. (2004). Norma Técnica Colombiana NTC-ISO 14001. Bogotá, Colombia: ICONTEC. Recuperado de: http://www.ins. gov.co/normatividad/Lineamientos $\% 20$ Sector/NTC-ISO\%2014001-2004.pdf?Mobile=1\&Source $=\% 2$ Fnormatividad $\% 2$ F_layouts $\% 2 F m o-$ bile\%2Fview\%2Easpx\%3FList\%3D9a3eeed1\%$252 \mathrm{Dadb} 1 \% 252 \mathrm{D} 4363 \% 252 \mathrm{D} 8458 \%$ 252Da3cd9d2b4835\%26View\%3D355f8e3f\%252 D6790\%252D4274\%252Db220\%

Instituto Colombiano de Normas Técnicas y Certificación, ICONTEC. (2007). Norma Técnica Colombiana NTC-OHSAS 18001. Bogotá, Colombia: ICONTEC. Recuperado de: http:// www.mincit.gov.co/mintranet/loader.php?lServicio $=$ Documentos \&IFuncion $=$ verPd $\mathrm{f} \& \mathrm{id}=67471$ \&name$=$ NTC-OHSAS18001.pdf\&prefijo=file

López-Celis, D. M., \& Peñalosa-Otero, M. E. (2017). La segmentación en la compra de productos socialmente responsables en Colombia y su impacto en el marketing. FACE: Revista de la Facultad de Ciencias Económicas y Empresariales, 17 (2), 6-14. 
Mora, V. (2007). Análisis de las Etapas Evolutivas de las empresas Familiares en la Ciudad de Florencia, Caquetá: Cinco Casos del Sector Comercial. (Tesis de Maestría). Florencia, Colombia: Universidad del Valle.

Morales-Rubianno, M. E., Ortiz-Riaga, C., Duque-Orozco, Y. V., \& Plata-Pacheco, P. A. (2017). Fuentes de conocimiento e imágenes de la innovación en micro y pequeñas empresas de turismo: agencias de viajes y hoteles en Bogotá y Pereira. Revista de Investigación, Desarrollo e Innovación, 7 (2), 217-230. doi: 10.19053/20278306.v7. n2.2017.6081

Muñoz-Saravia, A. (2007). Los Métodos Cuantitativo y Cualitativo en la evaluación de impactos en proyectos de inversión social. (Tesis doctoral). Recuperado de: http://www.eumed.net/tesis-doctorales/2007/ ams/index.htm

Organización Internacional para la Normalización -ISO. (2008). Norma Internacional ISO 9001. Ginebra:Translation Management Group.

Pérez, R. I., \& Ramírez, M. d. (9 de febrero de 2015). ¿Por qué fracasan las Pymes en Colombia?. Revista Dinero. Recuperado de: http://www.dinero.com/ economia/articulo/pymes-colombia/212958
Revista Dinero (14 de abril de 2016). Mipymes generan alrededor del $67 \%$ del empleo en Colombia. Recuperado de: https://www.dinero.com/edicion-impresa/pymes/articulo/evolucion-y-situacion-actual-de-las-mipymes-en-colombia/222395

Revista Semana (9 de mayo de 2015). Empresas de Familia: ¿Por qué fracasan?. Recuperado de: http:// www.semana.com/economia/articulo/empresas-familiares-por-que-no-sobreviven/441465-3

Sánchez, A., Hernández, T. J., Martínez, E., Villegas, E., \& García, C. (2018). Cultura organizacional en microempresas activadoras del desarrollo local. Margen: revista de trabajo social y ciencias sociales, 89 (6), 1-10.

Tagiuri, R., \& Davis, J. (1982). Bivalent attributes of the family firm. Family Business Review, 9, 107-119.

Vargas-Jiménez, I. (2012). La Entrevista en la Investigación cualitativa: nuevas tendencias y retos. Revista Calidad en la Educación Superior, 3 (1), 119139.

Vergara-Arrieta, J. J. (2015). Los sistemas de medición del desempeño estratégico frente a la competitividad y sustentabilidad. Saber, Ciencia y Libertad, 10 (2), 17-26. Recuperado de: https://dialnet.unirioja.es/servlet/articulo?codigo $=5295019$ 